\title{
Nasogastric tube induced refractory epistaxis during liver transplantation
}

Yun Hee Kim ${ }^{1}$, Byung Gun Lim² ${ }^{*}$, Yong Sang Yoon ${ }^{1}$, Soon Young Hong ${ }^{1}$, Yoon Sook Lee ${ }^{1}$, Woon Young Kim ${ }^{1}$, Jung Hyun Don ${ }^{1}$

1. Department of Anesthesiology and Pain Medicine, Korea University Ansan Hospital, Korea University College of Medicine, Ansan, Korea

2. Department of Anesthesiology and Pain Medicine, Korea University Guro Hospital, Korea University College of Medicine, Seoul, Korea

\begin{abstract}
Cirrhosis has many possible manifestations. These signs and symptoms may be either the direct results of liver cell failure or secondary to the resultant portal hypertension. Portal hypertension can decrease the number of platelets, which increases the risk of bleeding. Additionally, the liver plays a central role in hemostasis, because it is the site of clotting factors synthesis, coagulation inhibitors, and fibrinolytic proteins. Low platelet count and prolonged clotting times may increase the risk of epistaxis arising from minimal trauma, which can cause sometimes serious and occasionally fatal results. We experienced an intraoperative refractory epistaxis in a 60-year-old man with end-stage liver disease (ESLD) due to hepatitis B virusrelated hepatocellular carcinoma during liver transplantation. The patient started severe epistaxis after we attempted to place a nasogastric (NG) tube. We describe successful management of massive epistaxis in an operating room under appropriate anesthesia and close hemodynamic monitoring.
\end{abstract}

Keywords: End-stage liver disease; Epistaxis; Liver transplantation; Nasogastric tube

\section{Introduction}

Epistaxis from NG tube placement is rarely clinically severe in a normal population. But, it could be fatal in cases of abnormal coagulopathy. The risk factors, such as portal hypertension, coagulopathy, and endothelial dysfunction predispose ESLD patients to severe nasopharyngeal hemorrhage during NG tube placement (1). Camus $M$ et al. reported that most patients (80\%) had epistaxis after a recently placed NG tube that might have initiated or exacerbated pharyngeal bleeding, especially in patients with ESLD (2). In rare cases, this condition may lead to massive

\section{Corresponding author:}

Name: Byung Gun Lim, M.D., Ph.D.

Mailing Address: Department of Anesthesiology and Pain Medicine, Korea University Guro Hospital, 148 Gurodong-ro, Guro-gu, Seoul, Korea

Tel: +82-2-2626-3231 Fax: +82-2-26261438 E-mail: bglim9205@korea.ac.kr

Receive date: 2016-09-20| Accept date: 2016-10-15| Publish date: 2016-11-12

DOI: 10.7575/aiac.abcmed.16.04.04.04 
bleeding and even death. We report about management of the refractory epistaxis which could not be controlled by cauterization method under endoscopic guidance.

\section{Case Report}

A 60-year-old man (height $152 \mathrm{~cm}$, body weight $75 \mathrm{~kg}$ ) with end-stage liver disease (ESLD) due to hepatitis $B$ virus-related hepatocellular carcinoma was scheduled for a living donor liver transplant. Before surgery, he had liver cirrhosis (Child-Pugh score, 10; Model for End-stage Liver Disease [MELD] score, 14) accompanied by underlying diseases such as asthma. He had recurrent ascites, and mild esophageal and gastric varices. Initial blood gas and chemistry measurements were within the normal range. The platelet count was 59x $103 / \mu \mathrm{L}$, the international normalized ratio (INR) was 1.27, partial thromboplastin time was $35 \mathrm{~s}$ and fibrinogen was $104 \mathrm{mg} / \mathrm{dl}$.

Anesthesia was induced with intravenous propofol $120 \mathrm{mg}$ and rocuronium bromide 50 $\mathrm{mg}$ and maintained with a $50 \%$ oxygen/air mixture and desflurane 4 vol\% with continuous infusion of remifentanil and cisatracurium. During induction of general anesthesia, arterial cannulation was performed in the right radial artery and right femoral artery. A double-lumen $8 \mathrm{~F}$ dialysis catheter was inserted into the left internal jugular vein, $9 \mathrm{Fr}$ large bore catheter (Advanced Venous Access, Edwards Lifesciences, Irvine, CA, USA) and 7F triplelumen catheters were inserted into the right internal jugular vein. Hemodynamic monitoring included the right radial and femoral arterial, central venous pressures, and cardiac output measurements (Vigilance monitoring, Edwards Lifesciences, Irvine, CA, USA) using a radial artery catheter.

After anesthesia induction, we introduced a nasogastric (NG) tube before the surgery, we attempted to insert the tube into both nasal cavities due to insertion failure, and there was immediate bleeding from the mouth and noses. Repeated suction of the oral cavity was necessary, prompting examination of the nasopharynx by an otolaryngologist, which showed a venous bleeding point in the both posterior nasal cavities. We attempted to cauterize the bleeding sites under endoscopic guidance by an otolaryngologist, but we failed because of the bleeding tendency. At this time, total nasal blood loss was greater than $1500 \mathrm{ml}$. Furthermore, the nasal cavity bleeding increased because the anterior and posterior mucosa was eroded by endoscopy examination, and therefore, another technique was needed. We inserted an orogastric (OG) tube rather than the NG tube using video laryngoscopy. Next, we inserted both sides of nasal gauze packs with a fivefold dilution of $0.1 \%$ epinephrine $\mathrm{HCl}\left(1 \mathrm{mg} / \mathrm{ml}\right.$ Bosmin $^{\circledR}$, Jeil Pharm, Korea) to achieve haemostasis through compression and vasoconstriction effect, and oral gauze packs to drain blood (Figure. 1).

The liver transplantation (LT) surgery began approximately 95 minutes after induction of anesthesia. We changed the oral gauze packs when they filled with blood and simultaneously dripped a $0.02 \%$ epinephrine into the nasal gauze packs to keep effective epinephrine concentration levels during operation. The intervals between exchanging the oral gauze packs lengthened gradually. We used a fivefold dilution $(0.02 \%$, a total of $50 \mathrm{ml})$ of $0.1 \%$ epinephrine divided to five times during the operation. After we restored blood flow to the new liver, we constructed the biliary anastomosis, and by the end of surgery, there were no more blood stains on the oral gauze, and therefore, we decided to observe the progress of the patient.

Intraoperative final laboratory data showed that the platelet count was $95 \times 103 / \mu \mathrm{L}$, the international normalized ratio was 1.87 , partial thromboplastin time was $49 \mathrm{~s}$ and the fibrinogen levels were $101 \mathrm{mg} / \mathrm{dl}$. During the 
operation, the patient was given 15 units of packed red blood cells, 10 units of fresh frozen plasma, 3 units of plateletpheresis and 8 units of cryoprecipitate.

Sixteen hours later, the surgery ended, and the patient was transferred back to the ICU.
Without epistaxis during the post-operative course, he was transferred to the general ward on the second post-operative day in stable condition.

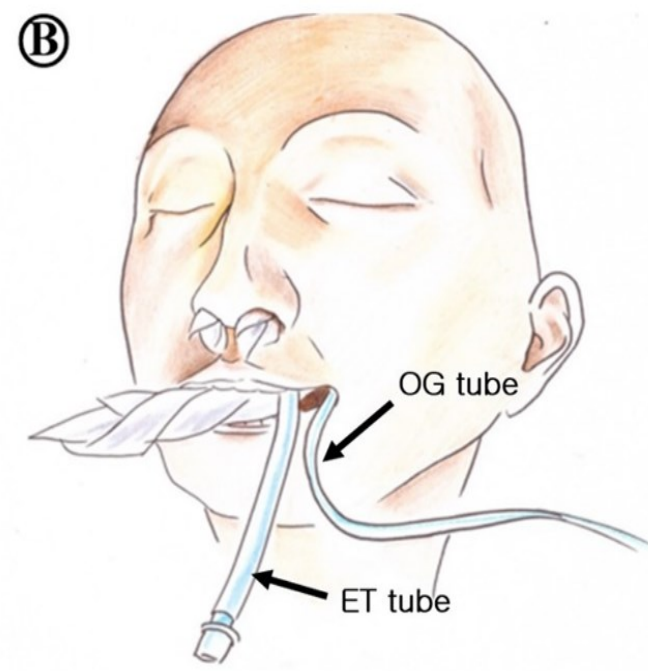

Figure 1: Orogastric tube was inserted instead of a nasogastric tube using video laryngoscopy. Next, both sides of nasal gauze packs with epinephrine were inserted to achieve haemostasis and oral gauze packs were inserted to drain blood. And additional diluted epinephrine was dripped into the nasal gauze packs to keep effective epinephrine concentration levels. (A) Sagittal view. (B) Frontal view. ET tube: endotracheal tube, OG tube: orogastric tube.

placement should be avoided as often as

\section{Discussion}

Epistaxis is classified as anterior or posterior depending on the source of bleeding, respectively, Kiesselbach's plexus or sphenopalatine arteries (3). Most cases of epistaxis are anterior which can be easily controlled. As in our case, most severe epistaxis is related to posterior nasopharyngeal epistaxis that may be occult, large volume, and associated with high mortality (4).

Epistaxis from NG tube placement occurs when the mucosa is eroded and vessels become exposed and subsequently broken. Abnormal coagulation may also increase the risk of epistaxis arising from minimal pharyngeal trauma. Therefore, NG tube possible, especially forceful or traumatic insertion, in patients with ESLD. Epistaxis in ESLD patients may be reduced by examining of the patient's nostril for septal deviation, applying a nasal vasoconstrictor, generously lubricating the tube tip, and using a gentle technique before the NG tube insertion. The tube should never be advanced against resistance. If gastric tube is required to patients with ESLD who was concerned about epistaxis, an OG tube rather than NG tube can be considered.

In patients with severe coagulopathy and hypofibrinogenemia, cryoprecipitate therapy is ideal. Transfusions of platelets are appropriate for patients with thrombocytopenia $1<$ $50 \times 103 / \mu \mathrm{L}$ ) associated with active bleeding or 
before invasive procedures in which a shortterm platelet count increase is noted. Infusion of fresh frozen plasma is more often effective and is recommended in patients with liver disease before invasive procedures or surgery because these, patients require transient correction in their prothrombin time $(2,5)$.

Once the diagnosis of refractory nasopharyngeal epistaxis is established, an otolaryngologist should be consulted about treatment options, which include nasal packing, applying a topical vasoconstrictive medication, possible embolization of arteries that supply that region, and/or endoscopic ligation with correction of coagulopathy $(6,7)$. Topical vasoconstrictors acting on alpha-adrenergic receptors in the nasal mucosa, cause vessels constriction, they are applied directly to the mucous membranes, where they will lead to prevention and cessation of the bleeding, or facilitate the exam in those that continue to bleed (8). Vasoconstrictive medications frequently used include phenylephrine, oxymetazoline, and epinephrine $(9,10)$. And, treatment of massive nasal bleeding involves careful volume resuscitation and administering an early combination of pharmacologic and endoscopy therapies.

We attempted to cauterize the bleeding sites under endoscopic guidance by an otolaryngologist, but failed. We could not postpone the surgery anymore. Therefore, immediately, the patient was managed by both nasal sides of antero-posterior gauze packing for compression and epinephrine for vasoconstriction to achieve hemostasis. Next, we planned to attempt other techniques such as arterial ligation or embolization that supplied that region at the end of the surgery.

Gauze, a Foley catheter, a nasal balloon catheter, or a nasal sponge/tampon can be used as a posterior pack. However, these balloons are easy to insert, but are not very effective in controlling posterior epistaxis (11).
Furthermore, in our case, the bleeding point could not be localized because bleeding points were widely originated anteriorly and posteriorly. Therefore, we used both sides of nasal gauze packing for compression. We performed bilateral nasal packing anticipating the direct pressing effect on the ongoing bleeding sites as usual, however we also expected indirect compressing force from the other side of the to cease bleeding more effectively. And additional diluted epinephrine was dripped into the nasal gauze packs to keep effective epinephrine concentration levels. To effective drain the blood and reduce oral mucosal injury by repeated oral suction inserted oral gauze packs. And then turning the patient onto his side and looking for nasal blood loss may help suggest an occult posterior pharyngeal hemorrhage as well as facilitate drainage. The oral gauze packs were changed new one when they soaked with blood. However, the nasal gauze packs did not change, because it might cause more damage and couldn't sustain compression in the nasal cavity.

In our case, no ongoing nasal bleeding was present by the end of surgery. There were two possible reasons for hemostasis: First, both sides of nasal gauze packs with epinephrine caused effective compression and vasoconstriction to stop bleeding. Second, it is known that an increase in fibrinolysis has been felt to be an important phenomenon responsible for bleeding during $L T$, usually diminishing within 60 in after graft reperfusion if the grafted liver begins to function (12). Therefore, in our case, although abnormal coagulation testing was seen in our patient, transplanted liver might restore impaired coagulation by a rebalanced hemostatic status.

In conclusion, NG tube placement can easily induce epistaxis because the nasal cavity has a large blood supply, and epistaxis cause sometimes serious and occasionally fatal 
results in ESLD patients. Therefore, a complete understanding of epistaxis that might occur during NG tube insertion is important for appropriate prevention and management, particularly in those patients with ESLD. Also, we suggest a management option for intractable multiple originated nasal bleeding in general anesthesia that is both sides of anteroposterior gauze packing for effective compression and dripping method of diluted epinephrine into the nasal gauze packs.

Acknowledgements: None

Conflicts of Interest: None

\section{References}

1. Tripodi A, Mannucci PM. The Coagulopathy of Chronic Liver Disease. New England Journal of Medicine. 2011;365:147-56.

2. Camus M, Jensen DM, Matthews JD, Ohning GV, Kovacs TO, Jutabha R, et al. Epistaxis in end stage liver disease masquerading as severe upper gastrointestinal hemorrhage. World Journal of Gastroenterology : WJG 2014;20:139938.

3. Koh E, Frazzini VI, Kagetsu NJ. Epistaxis: vascular anatomy, origins, and endovascular treatment. AJR Am J Roentgenol. 2000;174:845-51.

4. Gifford TO, Orlandi RR. Epistaxis. Otolaryngol Clin North Am. 2008;41:525-36.

5. Blonski W, Siropaides T, Reddy KR. Coagulopathy in liver disease. Curr Treat Options Gastroenterol. 2007;10:464-73.

6. Viehweg TL, Roberson JB, Hudson JW. Epistaxis: diagnosis and treatment. J Oral Maxillofac Surg. 2006;64:511-8.

7. Douglas R, Wormald PJ. Update on epistaxis. Curr Opin Otolaryngol Head Neck Surg. 2007;15:180-3.

8. Katz RI, Hovagim AR, Finkelstein HS, Grinberg Y, Boccio RV, Poppers PJ. A comparison of cocaine, lidocaine with epinephrine, and oxymetazoline for prevention of epistaxis on nasotracheal intubation. J Clin Anesth. 1990;2:16-20.

9. Groudine SB, Hollinger I, Jones J, DeBouno BA. New York State guidelines on the topical use of phenylephrine in the operating room. The Phenylephrine Advisory Committee. Anesthesiology. 2000;92:859-64.

10. Krempl GA, Noorily AD. Use of Oxymetazoline in the Management of Epistaxis. Annals of Otology, Rhinology \& Laryngology. 1995;104:704-6.

11. Paul J, Kanotra SP, Kanotra S. Endoscopic Management of Posterior Epistaxis. Indian Journal of Otolaryngology and Head \& Neck Surgery. 2011;63:141-4.

12. Garcovich M, Burroughs AK, Campanale C, Gasbarrini A. Monitoring of haemostasis during liver transplantation. Open Transplantation Journal. 2011;5:56-62. 